\title{
EQCM Measurement of Sn(II)/Sn Reaction in 1-butyl-1-methylpyrrolidinium bis(trifluoromethylsulfonyl)amide Room-temperature Ionic Liquid
}

\author{
Nobuyuki Serizawa, Naoki Tachikawa, Yasushi Katayama, ${ }^{*}$ and Takashi Miura \\ Department of Applied Chemistry, Faculty of Science and Technology, Keio University (Hiyoshi 3-14-1, Kohoku-ku, \\ Yokohama 223-8522, Japan)
}

Received February 19, 2009 ; Accepted June 20, 2009

\begin{abstract}
EQCM (Electrochemical Quartz Crystal Microbalance) measurement during Sn(II)/Sn electrode reaction in a roomtemperature ionic liquid was conducted using an impedance method EQCM, which can measure the resonance resistance and frequency simultaneously. The mass increased during Sn deposition and decreased during Sn dissolution. The viscosity and density of the electrolyte near the electrode, $\eta_{\mathrm{L}} \rho_{\mathrm{L}}$, can be estimated by monitoring the resonance resistance. The change of the $\eta_{\mathrm{L}} \rho_{\mathrm{L}}$ value during $\mathrm{Sn}(\mathrm{II}) / \mathrm{Sn}$ electrode reaction could be accounted for by the concentration profile of $\mathrm{Sn}(\mathrm{II})$ near the electrode.
\end{abstract}

Key Words : Ionic Liquid, Deposition, EQCM

\section{Introduction}

Several kinds of room-temperature ionic liquids have been investigated as the alternative electrolytes for metal electrodeposition since they have high cathodic stability and no proton source. Since ionic liquids containing bis(trifluoromethylsulfonyl)amide (TFSA-) have high stability against moisture compared with those containing other anions, ${ }^{1,2)}$ metal deposition has been studied widely in the TFSA-based ionic liquids. ${ }^{3,4)}$

Although EQCM is a useful tool for the analysis of electrodeposition, a conventional oscillator method EQCM (active method, self-excited) was difficult to be used in ionic liquids due to their high viscosity. ${ }^{5,6}$ However, metal deposition in ionic liquids has been studied successfully with an impedance method EQCM (passive method, separately-excited). ${ }^{7-13)}$ In this method, the resonance resistance and frequency are measured simultaneously. The resonance resistance reflects the product of viscosity and density of the electrolyte near the quartz crystal electrode. ${ }^{14)}$ In the present study, the change of the mass of the electrode and the change of the product of density and viscosity near the electrode during electrodeposition were examined using the impedance method EQCM.

The electrodeposition of Sn has been investigated from some ionic liquids. ${ }^{15-18)}$ In the TFSA--based ionic liquid, the charge transfer of $\mathrm{Sn}(\mathrm{II}) / \mathrm{Sn}$ reaction in BMPTFSA (BMP': 1-butyl-1-methylpyrrolidinium) was reported to be more facile than those of other M(II)/M (M = Mn, Fe, Co and $\mathrm{Zn}$ ) couples. ${ }^{15)}$ Therefore, a simple electrode reaction between Sn(II) and Sn in BMPTFSA was selected as a model system for the EQCM measurement in ionic liquids.

\section{Experimental}

1-butyl-1-methylpyrrolidinium bromide (BMPBr) was prepared by the reaction of 1-methylpyrrolidine (Tokyo
Kasei Kogyo, purity 98\%) and bromobutane (Tokyo Kasei Kogyo, 98\%) in acetonitrile (Junsei Chemical, $99.5 \%)$ at room-temperature, purified by recrystallization, and dried under vacuum at $120^{\circ} \mathrm{C}$ for 24 hours. BMPTFSA was prepared by interacting BMPBr with LiTFSA (Kanto Denka Kogyo, > $>9.9 \%$ ) in water, separated by extraction into dichloromethane (Junsei Chemical, $99.5 \%$ ) and finally dried under vacuum at $120{ }^{\circ} \mathrm{C}$ for 24 hours.

The anodic dissolution of Sn in BMPTFSA is a twoelectron-transfer reaction and its current efficiency is close to $100 \% .^{15)}$ Therefore, BMPTFSA containing 0.025 mol dm${ }^{-3} \mathrm{Sn}(\mathrm{II})$ was prepared by potentiostatic anodic dissolution with agitation using a two-compartment cell. Sn wire (Nilaco, 99.9\%) was used for the anode and cathode. Ag wire immersed in BMPTFSA containing $0.1 \mathrm{~mol}$ $\mathrm{dm}^{-3} \mathrm{AgCF}_{3} \mathrm{SO}_{3}$ (Aldrich, >99\%) separated from the ionic liquid with porous glass was used as a reference electrode ${ }^{19)}$. The cathode was placed in the separated compartment with a G4 glass filter.

Electrochemical measurements were conducted using a potentio/galvanostat (Hokuto Denko, HABF-501, 1510) with an EQCM system (SEIKO EG\&G, QCA922). Au, Pt and C-coated quartz crystal electrodes (SEIKO EG\&G, 9 $\mathrm{MHz}$, At-cut, $0.196 \mathrm{~cm}^{2}$ ) were employed as a working electrode. Sn wire (Nilaco, $99.9 \%$ ) was used as a counter and a reference electrode, respectively. Handling of hygroscopic materials and electrochemical measurements were carried out in an argon-filled glovebox with a continuous gas purification apparatus (Miwa Seisakusyo Co., Ltd., DBO-1KP-KO1). The concentration of $\mathrm{H}_{2} \mathrm{O}$ in the gas was kept under $1 \mathrm{ppm}$.

\section{Results and Discussion}

The frequency change, $\Delta f_{\mathrm{m}}$, is proportional to the mass change, $\Delta m$, as expressed by the Sauerbrey equation:20) 


$$
\Delta f_{\mathrm{m}}=-\frac{2 f_{0}^{2} \Delta m}{A\left(\mu_{\mathrm{q}} \rho_{\mathrm{q}}\right)^{1 / 2}}
$$

where $f_{0}$ is the resonance frequency, $A$ is the surface area of an electrode, $\mu_{\mathrm{q}}$ is the shear modulus of quartz, and $\rho_{\mathrm{q}}$ is the density of quartz. The total shift of the frequency $(\Delta f)$ is the sum of $\Delta f_{m}$ and $\Delta f_{\eta \rho}$, which is related to the viscosity, $\eta_{\mathrm{L}}$, and the density, $\rho_{\mathrm{L}}$, of the electrolyte near the electrode:21)

$$
\begin{gathered}
\Delta f=\Delta f_{m}+\Delta f_{\eta \rho} \\
\Delta f_{\eta \rho}=-f_{0}^{3 / 2} \sqrt{\frac{\eta_{\mathrm{L}} \rho_{\mathrm{L}}}{\pi \mu_{\mathrm{q}} \rho_{\mathrm{q}}}}
\end{gathered}
$$

The $\eta_{\mathrm{L}} \rho_{\mathrm{L}}$ value can be obtained from the resonance resistance, $R_{1}$, which is proportional to $\left(\eta_{\mathrm{L}} \rho_{\mathrm{L}}\right)^{1 / 2}$ with in a wide range of $\eta_{\mathrm{L}} \rho_{\mathrm{L}}$ value: ${ }^{14)}$

$$
R_{1}=\frac{A\left(2 \pi f_{0} \eta_{\mathrm{L}} \rho_{\mathrm{L}}\right)^{1 / 2}}{k^{2}}
$$

where $k$ is an electromechanical coupling factor. The resonance frequency and resistance changed immediately by ca. $-19 \mathrm{kHz}$ and $+2600 \Omega$, respectively, when an ionic liquid was dropped into a cell. The $k$ value can be estimated from the changes of the resonance frequency and the resonance resistance before and after contacting a liquid sample with a quartz electrode according to eqs. (3) and (4).

The cyclic voltammogram on a Pt electrode in 0.025 mol dm ${ }^{-3} \mathrm{Sn}$ (II)/BMPTFSA is shown in Fig. 1(a). Figure 1(b) shows the mass change during cyclic voltammetry, which was consistent with the deposition and stripping of $\mathrm{Sn}$ in the cyclic voltammogram, as shown in Fig. 1(a). The sensitivity of EQCM against the viscosity and density decays exponentially with the damping of the shear wave of the quartz through the distance from the electrode, the decay length, $\delta$, is defined by: ${ }^{21)}$

$$
\delta=\sqrt{\frac{\eta_{\mathrm{L}}}{\pi \rho_{\mathrm{L}} f_{0}}}
$$

In BMPTFSA, $\delta$ value is estimated to be $1.3 \mu \mathrm{m}$ from the viscosity and density of neat BMPTFSA $\left(\eta_{\mathrm{L}}=0.68 \mathrm{~g} \mathrm{~cm}^{-1}\right.$ $\mathrm{s}^{-1}$ and $\rho_{\mathrm{L}}=1.47 \mathrm{~g} \mathrm{~cm}^{-3}$ ). The $\eta_{\mathrm{L}} \rho_{\mathrm{L}}$ value is expected to depend on the average concentration of Sn(II) in the region of about $1.3 \mu \mathrm{m}$ thick from the electrode surface when the product of the viscosity and density is a function of the Sn(II) concentration. Figure 1(c) shows the change of $\eta_{L} \rho_{L}$ value during the potential sweep. The decrease in the $\eta_{L} \rho_{L}$ value during the cathodic sweep can be attributed to the drop in the Sn(II) concentration near the electrode by the cathodic reduction. The sharp increase in the $\eta_{\mathrm{L}} \rho_{\mathrm{L}}$ value during the anodic stripping, which is about $30 \%$ of initial $\eta_{\mathrm{L}} \rho_{\mathrm{L}}$ value, can be interpreted as the increase in the Sn(II) concentration by the anodic dissolution. Similar behavior was observed in $\mathrm{Ag}$ deposition and dissolution ${ }^{13)}$, and $\mathrm{Na}^{+}$doping and undoping in Nafion ${ }^{\circledR}$ film.2.

Almost identical behavior of $\Delta m$ and $\Delta \eta_{\mathrm{L}} \rho_{\mathrm{L}}$ was also observed when a gold or carbon electrode was used as

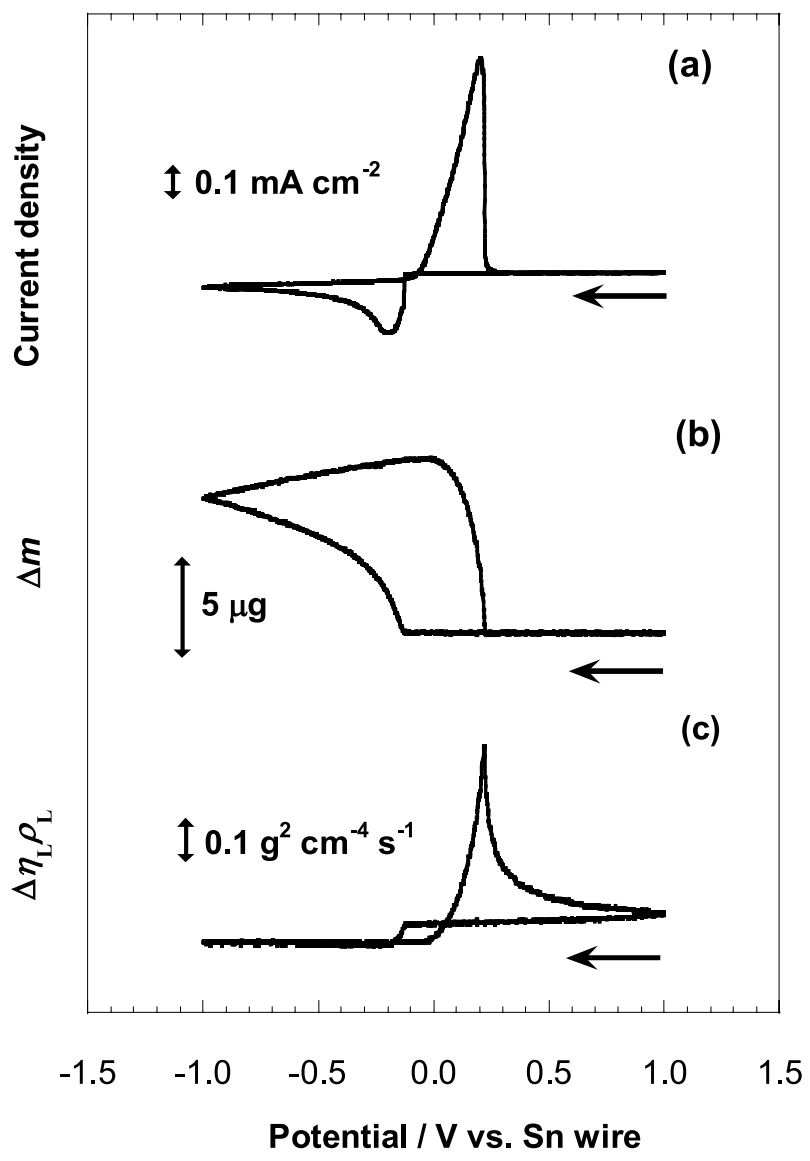

Fig. 1 (a) Cyclic voltammogram, (b) the mass change, and (c) the change of the $\eta_{\mathrm{L}} \rho_{\mathrm{L}}$ value in $0.025 \mathrm{M} \mathrm{Sn(II)/}$ BMPTFSA on a Pt electrode at $25^{\circ} \mathrm{C}$. Scan rate $: 2 \mathrm{mV} \mathrm{s}^{-1}$.

the working electrode. In case of a $\mathrm{Au}$ electrode, there were several anodic current peaks attributable to alloy formation. The XRD pattern of the Au electrode after the reduction of $\mathrm{Sn}$ (II) showed the diffraction peaks, which can be assigned to a Au-Sn alloy, AuSn.

Figure 2 shows the changes of the $\eta_{\mathrm{L}} \rho_{\mathrm{L}}$ value during potential step experiments. In the potential step in reducing direction, the $\eta_{\mathrm{L}} \rho_{\mathrm{L}}$ value decreased with the elapse of time and reached to a constant value, which is close to that of the neat BMPTFSA. It is expected that the average concentration of Sn(II) near the electrode decreases with the growth of diffusion layer and that it becomes constant when the growth of diffusion layer is suppressed by convection. In the potential step in oxidizing direction, the $\eta_{L} \rho_{L}$ value increased rapidly and then decreased with the elapse of time. The average concentration of Sn(II) near the electrode is expected to increase during the anodic dissolution of Sn. After all of $\mathrm{Sn}$ on the electrode is oxidized, the concentration near the electrode is considered to decrease with the diffusion of Sn(II) to the bulk. Thus, the monitoring the $\eta_{\mathrm{L}} \rho_{\mathrm{L}}$ value makes it possible to estimate the change of the concentration of the electroactive species near the electrode.

The change of the $\eta_{L} \rho_{L}$ value is detectable probably because the $\eta_{L} \rho_{L}$ value is significantly sensitive to the concentration of metal ions in the ionic liquid. This fea- 


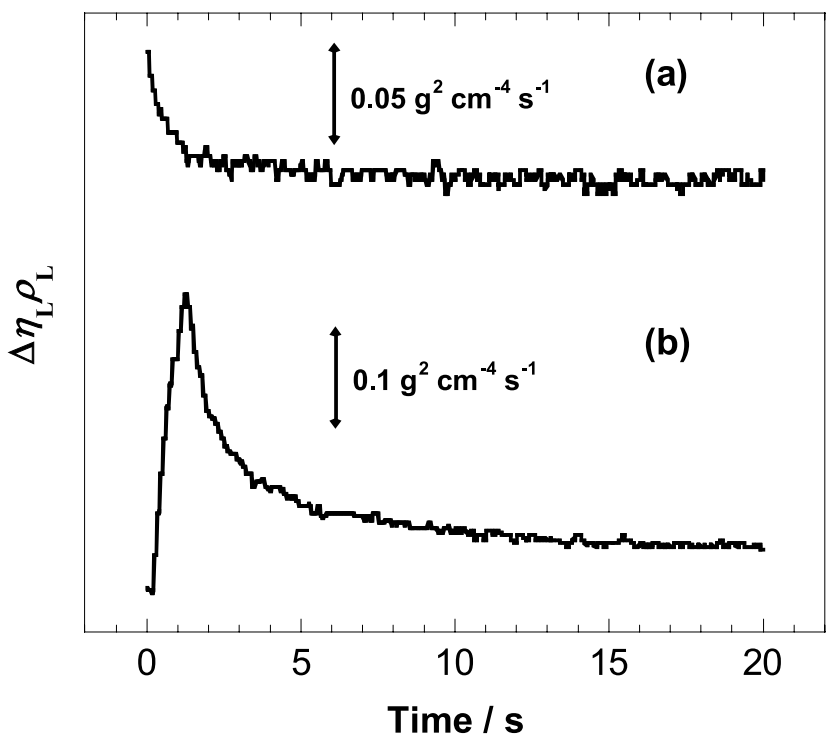

Fig. 2 The changes of the $\eta_{L} \rho_{L}$ value on a Au electrode during constant potential step experiments in $0.025 \mathrm{M}$ $\mathrm{Sn}$ (II)/ BMPTFSA at $25^{\circ} \mathrm{C}$; (a) deposition and (b) dissolution.

ture may be disadvantageous in the application of ionic liquids to various electrochemical devices. However, there is a possibility that ionic liquids can be used as electrolytic polishing baths of various metals. When a Sn electrode was dissolved anodically in BMPTFSA, the limiting current could be observed at relatively small over potentials. After the anodic dissolution, the surface of the Sn electrode was shiny and smooth. This observation is reasonably consistent with the $\mathrm{EQCM}$ results described above.

These results show that the impedance method EQCM can be a powerful tool for understanding the electrochemical processes in the ionic liquids.

\section{Conclusion}

The mass change was observed during Sn(II)/Sn electrode reaction in the ionic liquid using an impedance method EQCM. The change of the product of the viscosity and density near the electrode, $\eta_{\mathrm{L}} \rho_{\mathrm{L}}$, could be measured by monitoring the resonance resistance, $R_{1}$. It seems that the $\eta_{\mathrm{L}} \rho_{\mathrm{L}}$ value predominantly reflects the concentration of Sn(II) near the electrode. Thus, the concentration profile of the electroactive species near the electrode can be examined simultaneously with progress of an electrode reaction. However, this technique can be used for the systems, in which either reductant or oxidant is not soluble. Furthermore, the significant change of the $\eta_{L} \rho_{L}$ with the concentration of the electroactive species is required as seen in the ionic liquid.

\section{Acknowledgements}

The present work was financially supported by Grantin-Aid for Scientific Research on Priority Areas of "Science of Ionic Liquids" (No. 17073016) from the Ministry of Education, Culture, Sports, Science, and Technology, Japan (MEXT) and by Kato Foundation for Promotion of Science. The authors also thank Kanto Denka Kogyo for supplying LiTFSA.

\section{References}

1) P. Bonhôte, A. -P. Dias, N. Papageorgiou, K. Kalyanasundaram, and M. Grätzel, Inorg. Chem., 35, 1168 (1996).

2) D. R. MacFarlane, P. Meakin, J. Sun, N. Amini, and M. Forsyth, Phys. Chem. B, 103, 4164 (1999).

3) A. P. Abbott and K. J. McKenzie, Phys. Chem. Phys. Chem., 8, 4625 (2006).

4) S. Z. E. Abedin and F. Endres, ChemPhysChem, 7, 58 (2006).

5) H. Matsumoto, Z. -B. Zhou, H. Sakaebe, and K. Tatsumi, Electrochemistry, 73, 633 (2005).

6) J. J. Lee, Y. Mo, D. A. Scherson, B. Miller, and K. A. Wheeler, J. Electrochem. Soc., 148, C799 (2001).

7) A. Bund and E. Zschippang, 210th ECS Meeting Abstract, \#2042, Cancun, October 29-Nobember 3 (2006).

8) E. M. Moustafa, S. Z. E. Abedin, A. Shkurankov, E. Zschippang, A. Y. Saad, A. Bund, and F. Endres, J. Phys. Chem. B, 111, 4693 (2007).

9) F. Endres, S. Z. E. Abedin, A. Y. Saad, E. M. Moustafa, N. Borissenko, W. E. Price, G. G. Wallace, D. R. MacFarlane, P. J. Newman, and A. Bund, Phys. Chem. Chem. Phys., 10, 2189 (2008).

10) A. Ispas, A. Bund, F. Endres, 214th ECS Meeting Abstract, \#3055, Honolulu, October (2008).

11) N. Borisenko, S. Z. E. Abedin, and F. Endres, The Proceedings of 2008 Joint Symposium on Molten Salt, \#4B113, Kobe, October, (2008).

12) T. M. Benedetti, F. F. C. Bazito, E. A. Ponzio, and R. M Torresi, Langmuir, 24, 3602 (2008).

13) N. Serizawa, Y. Katayama, and T Miura, 214th ECS Meeting Abstract, \#3026, Honolulu, October (2008).

14) H. Muramatsu, E. Tamiya, and I. Karube, Anal. Chem., 60, 2142 (1988).

15) N. Tachikawa, N. Serizawa, Y. Katayama, and T. Miura, Electrochim. Acta, 53, 6530 (2008).

16) X. -H. Xu and C. L. Hussey, J. Electrochem. Soc., 140, 618 (1993).

17) G. Ling and N. Koura, Denki Kagaku (Electrochemistry), 65, 149 (1997).

18) J. -F. Huang and I. -W. Sun, J. Electrochem. Soc., 150, E299 (2003).

19) H. Ohno (Ed.), Electrochemical Aspects of Ionic liquids, p. 27, John Wiley \& Sons, Inc., Hoboken, NJ (2005).

20) G. Z. Sauerbrey, Z. Phys., 155, 206 (1959).

21) K. K. Kanazawa and J. G. Gordon II, Anal. Chem., 57, 1770 (1985).

22) H. Muramatsu, X. Ye, and T. Ataka, J. Electroanal. Chem., 347, 247 (1993). 